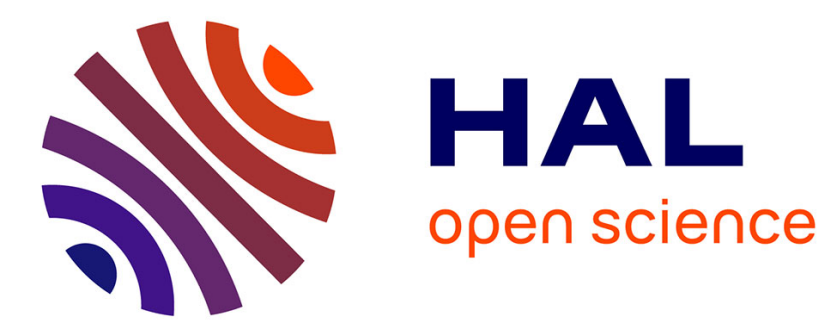

\title{
Environmental seasonality drives digital populations towards stable cross-feeding
}

Charles Rocabert, Carole Knibbe, Jessika Consuegra, Dominique Schneider, Guillaume Beslon

\section{- To cite this version:}

Charles Rocabert, Carole Knibbe, Jessika Consuegra, Dominique Schneider, Guillaume Beslon. Environmental seasonality drives digital populations towards stable cross-feeding. European Conference on Artificial Life (ECAL), Sep 2017, Villeurbanne, France. hal-01569093

\section{HAL Id: hal-01569093 \\ https://hal.science/hal-01569093}

Submitted on 26 Jul 2017

HAL is a multi-disciplinary open access archive for the deposit and dissemination of scientific research documents, whether they are published or not. The documents may come from teaching and research institutions in France or abroad, or from public or private research centers.
L'archive ouverte pluridisciplinaire HAL, est destinée au dépôt et à la diffusion de documents scientifiques de niveau recherche, publiés ou non, émanant des établissements d'enseignement et de recherche français ou étrangers, des laboratoires publics ou privés. 


\title{
Environmental seasonality drives digital populations towards stable cross-feeding
}

\author{
Charles Rocabert $^{1}$, Carole Knibbe ${ }^{1}$, Jessika Consuegra ${ }^{2,3}$, Dominique Schneider ${ }^{2,3}$ and Guillaume Beslon ${ }^{1}$ \\ ${ }^{1}$ Univ. de Lyon, CNRS, INRIA, INSA-Lyon, UCB Lyon 1, LIRIS UMR5205, F-69622 Lyon, France. \\ ${ }^{2}$ Univ. Grenoble Alpes, TIMC-IMAG, Grenoble, F-38000 Grenoble, France. \\ ${ }^{3}$ Centre National de la Recherche Scientifique (CNRS), TIMC-IMAG, F-38000 Grenoble, France. \\ guillaume.beslon@inria.fr
}

\begin{abstract}
Stable bacterial cross-feeding interactions, where one strain feeds on the waste of the other, are important to understand, as they can be a first step towards bacterial speciation. Their emergence is commonly observed in laboratory experiments using Escherichia coli as a model organism. Yet it is not clear how cross-feeding interactions can resist the invasion of a fitter mutant when the environment contains a single resource since there seems to be a single ecological niche. Here, we used digital organisms to tackle this question, allowing for detailed and fast investigations, and providing a way to disentangle generic evolutionary mechanisms from specificities associated with $E$. coli. Digital organisms with evolvable genomes and metabolic networks compete for resources in conditions mimicking laboratory evolution experiments. In chemostat simulations, although cross-feeding interactions regularly emerged, selective sweeps regularly purged the population of its diversity. By contrast, batch culture allowed for much more stable cross-feeding interactions, because it includes seasons and thus distinct temporal niches, thereby favoring the adaptive diversification of proto-species.
\end{abstract}

\section{Introduction}

Metabolic cross-feeding interactions between microbial strains are common in nature, and emerge during evolution experiments in the laboratory, even in homogeneous environments providing a single carbon source (Rosenzweig et al., 1994; Rozen and Lenski, 2000). In sympatry, when the environment is well-mixed, the reasons why emerging cross-feeding interactions may sometimes become stable and lead to monophyletic genotypic clusters occupying specific niches - named ecotypes (Cohan, 2002) - remain unclear.

As an alternative to evolution experiments in the laboratory, we developed $\mathrm{EVO}^{2} \mathrm{SIM}$ (http://www.evoevo.eu/ evo2sim-software/), a multi-scale model of in silico experimental evolution (ISEE, Hindré et al. 2012), equipped with the whole tool case of experimental setups, competition assays, phylogenetic analysis, and, most importantly, allowing for evolvable ecological interactions. Digital organisms with an evolvable genome structure encoding an evolvable metabolic network evolved for tens of thousands of genera- tions in environments mimicking the dynamics of real controlled environments, including chemostat or batch culture, providing a single limiting resource.

By performing in silico evolution experiments, we studied the environmental conditions in which a stable cross-feeding interaction could occur. To this aim, we let populations of digital organisms evolve in three different environments:

(1) Seasonal. In this environment, the organisms grow on a single resource, periodically provided each $\sim 7$ generations, the rest of the environment being rinsed at the same time. The seasonal environment mimics the single carbon source serial transfer setup of the Long Term Evolution Experiment (LTEE, Rozen and Lenski 2000);

(2) Continuous. Here, the digital organisms grow on the same single resource, but continuously provided. The periodic rinse is replaced by a small degradation of the free metabolites in the environment (exogenously provided or released by the organisms). The continuous environment mimics a single carbon source chemostat setup.

(3) Poisson. This environment is exactly the same as the seasonal one except that the serial passages are no more periodic. The resource is provided (and the environment is rinsed) at random times following a Poisson law.

We evolved 12 independent populations in each of the three types of environment. Importantly, the total amount of resource available is the same in the three environments.

\section{Results}

Comparison of the evolutionary outcome in the three environments shows important differences in the structure of the populations (see Fig. 1). Results show that the longterm maintenance of cross-feeding interactions is strongly favored in the seasonal environment. In this environment, half of the simulations evolved a stable cross-feeding interaction, with two monophyletic ecotypes coexisting via a negative frequency-dependent interaction. At each cycle, a first ecotype grows during the first season, feeding on the primary resource and releasing by-products, while 
the second ecotype exclusively feeds on by-products during the second season. The stable coexistence of both ecotypes is based on niche construction, followed by a negative frequency-dependent interaction, as the $\mathrm{S}$ and $\mathrm{L}$ ecotypes in the LTEE (Rozen and Lenski, 2000). According to our model, batch culture experiments seem to especially favor the evolution of stable cross-feeding polymorphisms owing to the cyclic nature of the environment that generates the conditions for the existence of at least two stable seasons: a first season is externally generated by the cyclic mechanism (thus being intrinsically stable) while the second one is generated by the replacement of the exogenously-provided nutrient by the secreted by-products through a mechanism of niche construction.

In the continuous environment, where the primary resource is constantly provided (like in a chemostat), crossfeeding interactions emerged, but were not stable because of competitive exclusion. In this case, organisms enriched their environment via their metabolic activity, such that mutants were temporarily able to feed on by-products. But the absence of seasonality precludes any possibility for the stabilization of cross-feeding interactions.

Our multi-scale model allowed us to investigate the impact of resource dynamics on the organization of genome (e.g., gene amplification) and of the metabolic network, and to dissect the precise mechanism behind the evolved robustness of the cross-feeding interaction in the seasonal environment. We also demonstrated that those results are robust to model parameters variation. Indeed, stable cross-feeding interactions emerged in the periodic environment for a wide range of parameter values, including well-mixed populations and infinite diffusion rate, while they never appeared in the continuous environment, thus reinforcing our conclusions.

As a conclusion, the stability of a cross-feeding polymorphism should only be analyzed in the light of the robustness of each ecotype against selective sweeps by other ecotypes (Cohan, 2002). This mechanism is observed in the LTEE, as well as in our model. Such complex interactions between external factors, emergent cross-feeding interactions and niche construction are therefore of primary importance to understand the evolution of microbial communities in well-mixed environments. Using a computational model of ISEE to decipher those interactions seems to be a rich complementary approach to wet experiments and mathematical modeling.

This work has been published in the PLoS Computational Biology journal (Rocabert et al., 2017).

\section{Acknowledgements}

This work was supported by the European Commission 7th Framework Programme (FP7-ICT-2013.9.6 FET Proactive: Evolving Living Technologies) EvoEvo project (ICT610427, http://www.evovo.eu/). The authors thank all the partners of the EvoEvo project for fruitful discussions.

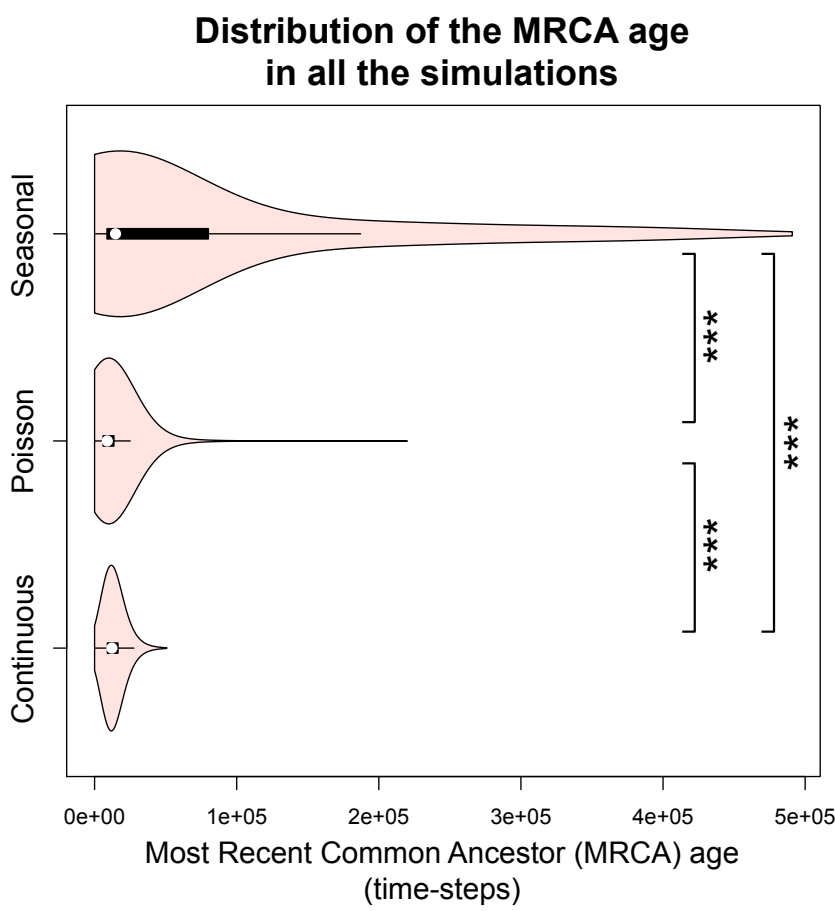

Figure 1: Distribution of the Most Recent Common Ancestor age in all the simulations. For each environment, we computed the distribution across repetitions of the Most Recent Common Ancestor (MRCA) age, for each simulation time-step. All pairwise Student tests are significant, with Bonferroni correction ( $\mathrm{p}$-value $<0.001 / 3$ ). In the seasonal environment, deepest trees are also the ones that exhibit two well differentiated ecotypes (while this is not the case in the Poisson and continuous environments, not shown here).

\section{References}

Cohan, F. M. (2002). What are bacterial species? Annual Reviews in Microbiology, 56(1):457-487.

Hindré, T., Knibbe, C., Beslon, G., and Schneider, D. (2012). New insights into bacterial adaptation through in vivo and in silico experimental evolution. Nature Reviews Microbiology, 10(5):352-365.

Rocabert, C., Knibbe, C., Consuegra, J., Schneider, D., and Beslon, G. (2017). Beware batch culture: Seasonality and niche construction predicted to favor bacterial adaptive diversification. PLOS Computational Biology, 13(3):e1005459.

Rosenzweig, R. F., Sharp, R., Treves, D. S., and Adams, J. (1994). Microbial evolution in a simple unstructured environment: genetic differentiation in escherichia coli. Genetics, 137(4):903-917.

Rozen, D. E. and Lenski, R. E. (2000). Long-term experimental evolution in escherichia coli. viii. dynamics of a balanced polymorphism. The American Naturalist, 155(1):24-35. 